\title{
ARTICLE OPEN \\ Inkjet-printed MXene micro-scale devices for integrated broadband ultrafast photonics
}

\author{
Xiantao Jiang ${ }^{1,2,5}$, Wenjia $\mathrm{Li}^{2}$, Ting $\mathrm{Hai}^{3}$, Rui Yue ${ }^{2}$, Zhangwei Chen ${ }^{4}$, Changshi Lao ${ }^{4}$, Yanqi Ge ${ }^{1,2}, \mathrm{Guoqiang} \mathrm{Xie}^{3}$, Qiao Wen $\mathbb{E}^{2}$ and \\ Han Zhang (D)
}

MXene, as a novel 2D crystal material, possessing tunable bandgap, low optical attenuation and broadband nonlinear optical responses that may promote the fabrications of advanced electro-photonics devices has gathered remarkable attention recently. However, current investigations of 2D crystals for photonics devices suffer from the limitations of reproducibility, scalability, and compatibility. Inkjet printing is one of the powerful additive manufacturers that facilitate well-controlled, low-cost, scalable and small-footprint electrophotonics devices on myriad substrates. Herein, we directly inkjet printed MXene nanosheets in laser resonators with both fiber and free-space geometrics, and achieved extensive spectral band ultrafast laser operations from near- to the mid-infrared regime with pulse duration going to 100 femtoseconds. The demonstrations of versatile inkjet-printed devices based on MXene, while forthputting its distinct electro-optical properties, may allow the realizations of advanced MXene enable photonics devices shortly.

npj 2D Materials and Applications (2019)3:34 ; https://doi.org/10.1038/s41699-019-0117-3

MXene, ${ }^{1}$ as a glaring multi-feature two-dimensional (2D) crystal, has recently experienced a rapid proliferation of interests from energy storage $^{2}$ medical therapy ${ }^{3}$, and imaging, ${ }^{4}$ electromagnetic interference (EMI) shielding, ${ }^{5}$ catalyst, ${ }^{6}$ sea water purification, ${ }^{7}$ ultrafast photonics, ${ }^{8}$ to only name a few. MXene represents a big family with the chemical composition of $M_{n+1} X_{n} T_{x}$, where $M$ is an early transition metal, $\mathrm{X}$ is $\mathrm{C}$ and/or $\mathrm{N}, \mathrm{T}$ is the surface terminations $(-\mathrm{OH}$, $-\mathrm{O}$ or $-\mathrm{F}$ ), and $n=1,2$, or 3 . The chemical richness and $2 \mathrm{D}$ atomical layer structure of MXene equip themselves with variable and tailorable physical and chemical properties. Specifically, the large bandgap tunability, ${ }^{9-14}$ a suggested lower optical attenuation ${ }^{15,16}$ compared to the well-known graphene $2.3 \%$ per atomical layer, ${ }^{17}$ and the broadband $(0.8-1.8 \mu \mathrm{m})$ nonlinear optical responses ${ }^{8}$ of MXene indicate the huge potential to be taken for efficiently light manipulations. However, there have been only a few attempts to utilize the excellent optical properties for plasmonics ${ }^{18}$ and ultrashort laser generations from 1.06 to $1.88 \mu \mathrm{m}^{8,19-21}$ through drop cast or spin-coating. Yet, these techniques suffer from the bottlenecks of reproducibility, scalability, and compatibility. MXenes can be available either via top-down acid etching method $^{1}$ or through bottom-up CVD approach. ${ }^{22}$ The previous one overwhelming the other approaches in the aspects of feasibility, yielding, controllability and finance efficiency. Thus, the fine-controlled solution processed techniques are reckoned as the primary way to utilize MXene for device fabrications.

Inkjet printing is a forefront technique to exploit the merits of $2 \mathrm{D}$ materials for printed optoelectronic devices with small-footprint, integration, substrates/geometrics compatibility, scalability, and low cost. Indeed, a large variety of 2D materials, including reduced graphene oxide, ${ }^{23-28}$ graphene, ${ }^{29-42}$ transition metal dichalcogenides, $31,34,38,39,42,43$ boron nitride, ${ }^{31,34,40-42}$ as well as black phosphorus ${ }^{44}$ have been incorporated into the printing inks to construct electrodes, photodetectors, gas/strain sensors, transistors, supercapacitors, photovoltaic devices, read-only memories, EMI shield and so forth. Success inkjet-printed pattern realization is the interplay result of substrates, ink rheological properties and printer parameters. ${ }^{45,46}$ The choosing of ink solutions is regarded as the key point. High viscosity solution like N-methyl-2-pyrrolidone (NMP) and dimethyl sulfoxide (DMSO) are normally used in the 2D material liquid exfoliation processes, and thus can be directly used for ink formulation. However, high-temperature annealing after-treatment may be incurred to remove the polymers which will impair the performance of 2D crystals. $^{29,31,38-40}$ Single or mixed low boiling point alcohols (e.g., ethanol, 2-butanol, isopropanol) and biological compatible water are also proposed as the ink solutions with careful ink surface tension and viscosity engineering. ${ }^{23,30-34,37,41,42} \mathrm{~A}$ small amount of polymers or proteins can also be added into the solutions that act as "binders" to improve the printed film uniformity. Very recently, the viable of printed MXene has been demonstrated with a DMSO + protein formulated ink, where a large diameter nozzle $(125 \mu \mathrm{m})$ is required, and thus the spatial pattern resolution is limited to $120 \mu \mathrm{m}^{47}$

In this paper, metallic titanium carbide $\mathrm{Ti}_{3} \mathrm{C}_{2} \mathrm{~T}_{x}$ MXene is deliberately chosen and prepared via acid etching approach. Low boiling point, non-toxic, "binder-free" MXene nanosheets ink is formulated using isopropanol (IPA, boiling point $82.6^{\circ} \mathrm{C}$ ) for establishing functional devices on silica glass, $\mathrm{SiO}_{2} / \mathrm{Si}$ wafer, sidepolished fiber, flexible and transparent PET film, and gold mirror

\footnotetext{
${ }^{1}$ Shenzhen Engineering Laboratory of Phosphorene and Optoelectronics, Collaborative Innovation Center for Optoelectronic Science and Technology, College of Optoelectronic Engineering, Shenzhen University, Shenzhen 518060, China; ${ }^{2}$ Key Laboratory of Optoelectronic Devices and Systems of Ministry of Education and Guangdong Province, College of Optoelectronic Engineering, Shenzhen University, Shenzhen 518060, China; ${ }^{3}$ Key Laboratory Laser Plasmas, Collaborative Innovation center of IFSA (CICIFSA), School of Physics

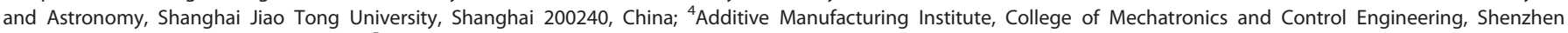
University, Shenzhen 518060, China and ${ }^{5}$ College of Chemistry and Environmental Engineering, Shenzhen University, Shenzhen 518060 , China

Correspondence: Guoqiang Xie (xiegq@sjtu.edu.cn) or Qiao Wen (wenqiao@szu.edu.cn) or Han Zhang (hzhang@szu.edu.cn)

These authors contributed equally: Xiantao Jiang, Wenjia Li
}

Received: 27 March 2019 Accepted: 26 July 2019

Published online: 04 September 2019 
a

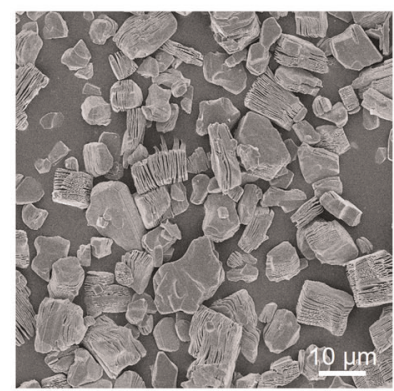

d

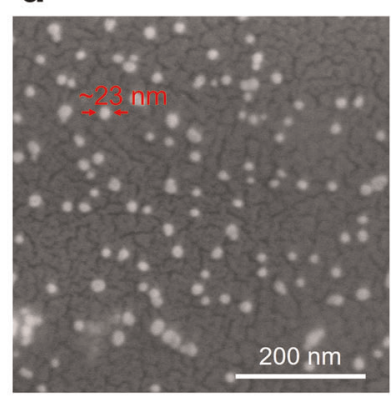

h

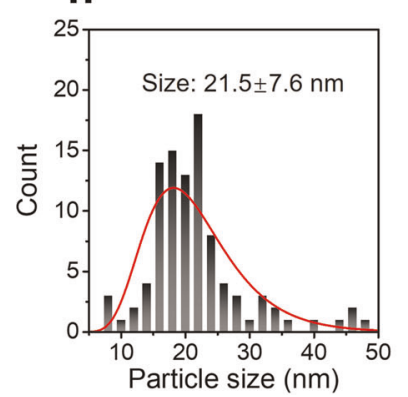

b

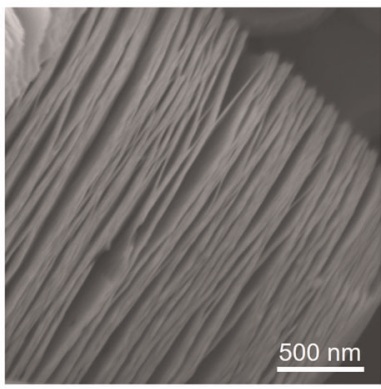

e



i

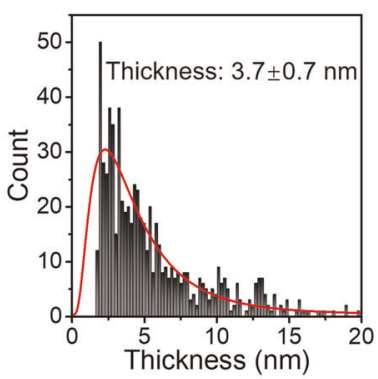

C

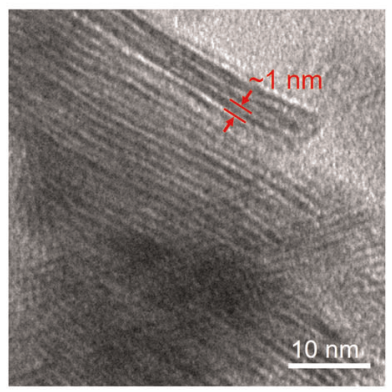

f

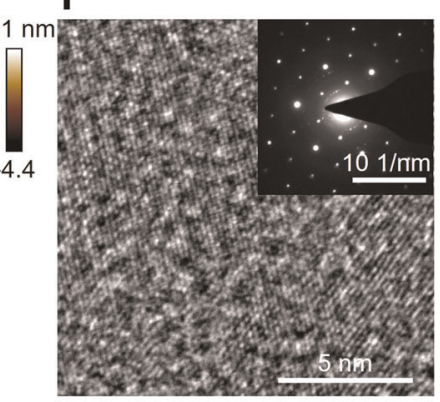

g

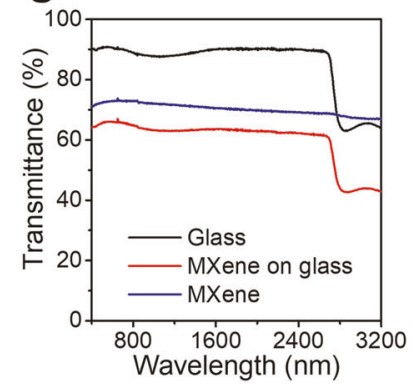

Fig. 1 Morphological characterizations of MXene. a SEM image of the prepared MXene powder via acid etching technique. $\mathbf{b}$ The zoom-in SEM image of MXene. c HRTEM image shows the interlayer thickness of MXene. d SEM and e AFM image of the MXene nanosheets in the IPA ink precursor. $\mathbf{f}$ HRTEM image of MXene nanosheets; inset: the corresponding SEAD pattern. $\mathbf{h}$ Size (Feret's diamter) and $\mathbf{i}$ thickness distributions of the nanosheets characterized via SEM and AFM, respectively. $\mathbf{g}$ The optical transmission of MXene nanosheets deposited on a silica glass substrate

substrates. To advance the utilize of the broadband nonlinear optical responses of MXene, the printed side-polished fiber and gold mirror have been integrated to both fiber and solid-state laser resonators, to achieve ultrafast laser operations covering the optical spectrum of $1-3 \mu \mathrm{m}$. Mode-locking operation with pulse duration going to $100 \mathrm{fs}$ has been achieved at the telecommunication window, while Qswitched lasers have been demonstrated in solid-state resonators and in the mid-infrared regime. The virtue of the demonstrations not only lies on broadband ultrafast laser generations via versatile printed MXene photonic devices but are also anticipated to point out a methodology for complex laser resonators integration with functional 2D crystal materials.

\section{RESULTS AND DISCUSSION}

Ink formulation

In this contribution, titanium carbide $\left(\mathrm{Ti}_{3} \mathrm{C}_{2} \mathrm{~T}_{x}\right)$ MXene was prepared via feasible acid etching method, as described in Ref. ${ }^{8}$ The delaminated $\mathrm{Ti}_{3} \mathrm{C}_{2} \mathrm{~T}_{x}$ was then washed by de-ionic water and dried. The morphology of the prepared MXene is shown in Fig. 1a, b, where typical accordion-like structures can be clarified, confirms the good extraction of $\mathrm{Al}$ atoms from its corresponding MAX phase $\mathrm{Ti}_{3} \mathrm{AlC}_{2}$. The atomic lattice was characterized to be hexagonal space group $\mathrm{P}_{3} / \mathrm{mmc}$ (Fig. 1f) with an interlayer distance of $\sim 1 \mathrm{~nm}$ (Fig. 1c), which in agreement with the theoretical predictions. ${ }^{48}$ The particle size of the obtained MXene powder after acid etching procedures is around 10-30 $\mu \mathrm{m}$ (see Fig. 1a), which can result in nozzle clogging during the inkjet printing. Typically, the particle size should be smaller than 1/50 the of the nozzle diameter in order to avoid nozzle choking. ${ }^{29,49}$ Therefore, the MXene particle size is required to be smaller than $400 \mathrm{~nm}$ when using a $22 \mu \mathrm{m}$ nozzle. To reduce the particle size, the prepared MXene powder was firstly dispersed into isopropyl alcohol (IPA) solution with a concentration of $5 \mathrm{mg} / \mathrm{ml}$ and then underwent violent ultrasonic bath in ice-cooled water over $72 \mathrm{~h}$. After this, a home-made vacuum filtration apparatus with a polytetrafluoroethylene (PTFE) membrane (pore size $\sim 220 \mathrm{~nm}$ ) was used to separate the produced MXene nanosheets into a flask. The mean particle size (Feret's diameter) and thickness of the MXene nanosheets in the IPA solution were checked via SEM and AFM to be $21.5 \pm 7.6 \mathrm{~nm}$ and $3.7 \pm 0.7 \mathrm{~nm}$, respectively, well satisfy the particle size requirements of printing (see Fig. 1d, e). However, we found the concentration of MXene nanosheets is rather low after separation for efficiently pattern fulfillment, even though multiple passes are applied. The MXene nanosheets IPA solution was then concentrated via a rotavapor to formulate the ink. The 
concentration of the MXene ink was characterized to be $\sim 2 \mathrm{mg} /$ $\mathrm{ml}$. Previously, efficient inkjet-printed devices have been reported using an ink concentration as low as tens $\mu \mathrm{g} / \mathrm{ml}$ in different solutions, ${ }^{31}$ indicating that the concentration of the materials is not a stringent prerequisite. However, a higher ink concentration features synergistic advantages to the final productions, such as reduces the printing time, higher uniformity due to the material pinning effect that effectively avoids the material carry during the ink drying, alleviates the re-dispersion. The optical attenuation of MXene ink drop cast on a silica glass is presented in Fig. 1g, which shows an impartial absorption from 400 to $3000 \mathrm{~nm}$, agrees well with its zero bandgap (or $E_{\mathrm{g}}<0.2 \mathrm{eV}$ ) electronic band structure, ${ }^{1}$ and indicating the broadband nonlinear optical modulation spectral window.

The devices pattern is constructed via the deposition of multiple single ink droplets that determine the print pixel resolution, thus, the engineering of the single droplet is of crucial for the device fabrications. Stable ejecting of single droplets from a given nozzle can be qualitatively estimated by the inverse Ohnesorge number $Z=\frac{\sqrt{\rho \gamma d}}{\eta}$, where $\rho, \gamma$, and $\eta$ represents the ink density, surface tension and viscosity respectively, $d$ is the diameter of the nozzle. An unitless value of $Z$ between 1 and 14 suggests a stable single droplet ejecting. Ink with $Z$ value smaller than 1 is hard to be ejected, while with $Z$ larger than 14 tend to generate satellite droplets. ${ }^{46,50}$ A brief summary of rheological properties of the the formulated 2D materials ink is presented in Table 1. The obtained MXene ink features a density of $0.79 \mathrm{~g} / \mathrm{cm}^{3}$, surface tension of $23 \mathrm{mN} / \mathrm{m}$, viscosity of $2.4 \mathrm{mPas}$, thus giving $Z \sim 8.3$, which is similar to recently reported ink based on black phosphorus ${ }^{44}$ and h-boron nitride. ${ }^{40}$ The prepared $M X e n e$ nanosheets ink can stable for more than three months in a fridge, without particle aggregation and ink stratification were observed. This might be due to the high negative zeta potential of MXene nanosheets. ${ }^{51}$ A stable ejected droplet sequences observed from the printer stroboscopic camera is presented in Fig. 2b. The droplet is further required to wet the substrate to form a continous coating though the ink is suitable for stable ejecting. ${ }^{45}$ Previous reports suggest the ink surface tension should be $7-10 \mathrm{mN} / \mathrm{m}$ lower than the substrate surface energy. ${ }^{44}$ The asprepared MXene ink is suitable for substrates including silicate glass, $\mathrm{SiO}_{2} / \mathrm{Si}$ wafer $\left(\mathrm{SiO}_{2} \sim 36 \mathrm{mN} / \mathrm{m}^{52}\right)$, and polyethylene terephthalate (PET, $\sim 48 \mathrm{mN} / \mathrm{m}^{53}$ ). All of them have been applied without any surface treatments in this project to confirm the ink's wide adaptability.

After printing, the droplet drying process is another key point for the final device quality. The higher evaporation ratio at the outer edge of the droplets could lead to the outward capillary flows that carry the material to the external edge and create the well-known non-uniform "coffee ring" effect. To avoid the "coffee ring" effect, several strategies have been proposed, including mixed solutions with different surface tensions to form the reverse Marangoni flows, low surface tension to achieve high-level wetting, large particle size, high ink concentration or polymer/ protein binders to "freeze" the particles, low solution evaporation temperature to mediate the evaporation ratio difference, to demonstrate well-printed functional devices. ${ }^{30,35,54,55}$ Herein, IPA with low surface tension and low evaporation temperature $\left(82.6^{\circ}\right.$ C) was chosen as the ink solution, and the dispersed MXene nanosheets in that IPA solution was concentrated to alleviate the printed pattern non-uniformity. Through these means, negligible "coffee ring" can be observed experimentally on both the substrates (see Fig. 2 and Supplementary Information).

\section{Device fabrications}

The ability of inkjet printing to form arbitrary 2D or quasi-3D patterns via the deposition of single ink droplets has significantly promoted the explorations of advanced micro electro-optical devices based on two-dimensional crystals. ${ }^{31,37,39,40,42}$ Previous studies indicate that MXene can be a promising broadband saturable absorber from visible to the mid-infrared regime with linear optical losses as low as $\sim 1 \% / \mathrm{nm} .^{8,16,19}$ However, the integration to the laser resonators and the finely controlled deposition thickness, geometrics, and dimensions remain been barely touched to date. Hence, we adopted a side-polished fiber and a gold mirror as the substrates to form the saturable absorber devices in pursuit of integration with different laser resonator setups. In the printing process (DMP-2850, FUJIFILM Dimatix), the substrate temperature is $50^{\circ} \mathrm{C}$, the voltage for the nozzle actuator is $18-23 \mathrm{mV}$ depends on the nozzle chosen, the tickle frequency is $23 \mathrm{kHz}$. By altering the inter-droplet distance, different patterns such as dots matrix, stripes and be printed as required (see Fig. 2d, e). Decreasing the inter-droplet distance to $35 \mu \mathrm{m}$, uniform and continuous MXene nanosheets film can be obtained on the substrates. We found multi-passes could help to improve the film uniformity due to the small inter-droplet distance. A range of printed layers on both side-polished fiber $(4 \mathrm{~L}, 6 \mathrm{~L}, 8 \mathrm{~L}, 10 \mathrm{~L}, 12 \mathrm{~L}$; L: layer) and gold mirror ( $4 \mathrm{~L}, 8 \mathrm{~L}, 10 \mathrm{~L}, 16 \mathrm{~L}, 20 \mathrm{~L}$ ) samples were prepared to engineer the saturable absorption properties for particular resonators. There are a $5 \mathrm{~min}$ break every two passes for drying on the side-polished fiber (as well as glass, PET, $\mathrm{SiO}_{2} / \mathrm{Si}$ wafer), while for the thicker gold mirror substrate, the drying time could last about more than $10 \mathrm{~min}$ at $60^{\circ} \mathrm{C}$. Subtle stacking trace and white light interference colors can be recognized from Fig. $2 f$ k. Note, the printing technique can be agilely transformed on the substrates of glass, transparent and flexible PET film, silica/silicon wafer for specified applications (see Supplementary Information). Figure 2c shows a logo of "Shenzhen University" that been printed on a transparent and flexible PET film via using the MXene nanosheets ink.

\section{Laser performance}

The investigation of ultrafast lasers with high electric field amplitude, short temporal duration and high repetition rate, driven by the wide application demands in both civil and military industries has found fruitful achievements via semiconductor saturable absorber mirror (SESAM). ${ }^{56}$ However, the sophisticated fabrication processes and the limited frequency bandwidth of SESAM encounters the barriers of high financial cost, low compatibility, and narrow modulation bandwidth. Alternatives based on low dimensional materials, such as graphene, carbon nanotubes (CNTs), topological insulators, transition metal dichalcogenides, and epsilon-near-zero (ENZ) medium have recently been proposed, ${ }^{57,58}$ with which broadband optical modulation capability, low optical loss, high modulation depth and low bleaching threshold have been demonstrated. Even though, the exploiting of broadband saturable absorbers, especially for the mid-infrared laser sources based on low-dimensional materials is far for closure, in the aspects of fabrication technologies, materials, resonator geometrics, and optical mechanisms.

To demonstrate the broadband optical amplitude modulations and wide adaptability of the printed MXene saturable absorbers, four laser resonators with different operating wavelengths and configurations were designed and established. Figure $3 a$, b shows the architecture of all-fiber ring resonators with ytterbium- and erbium-doped fiber as the gain medium. The length of the $\mathrm{Yb}$ doped $(250 \mathrm{~dB} / \mathrm{m} @ 980 \mathrm{~nm}, 8 / 125 \mu \mathrm{m})$ and Er-doped $(4.45 \mathrm{~dB} / \mathrm{m}$ $@ 980 \mathrm{~nm}, 8 / 125 \mu \mathrm{m})$ fiber are 1 and 4 meters, results in cavity length of 12.7 and 17.8 meters, respectively. The ring oscillator consists of a laser diode (LD, $980 \mathrm{~nm}, P \max =700 \mathrm{~mW}$ ) as the pump, a wavelength division multiplexer (WDM) for input coupling, the impurified gain medium, a polarization controller $(\mathrm{PC})$, a polarization independent isolator (PI-ISO), an optical coupler (OC, 10\%), and the side-polished fiber saturable absorber. 


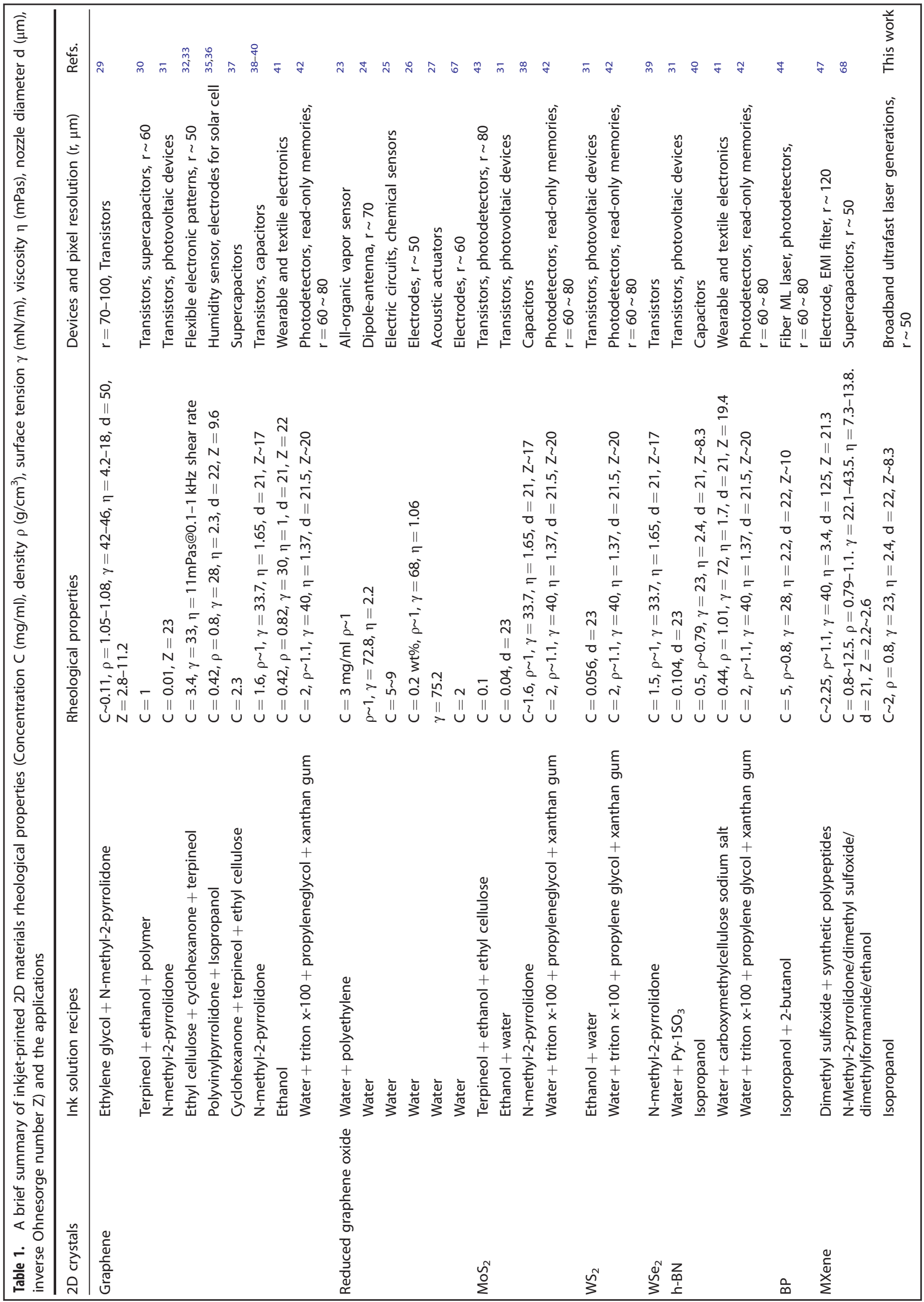


a

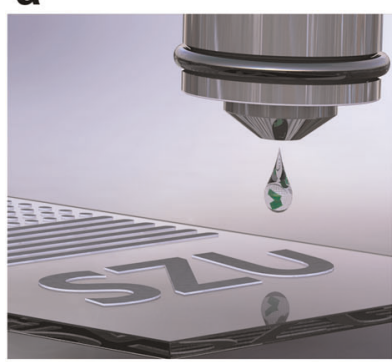

d



h

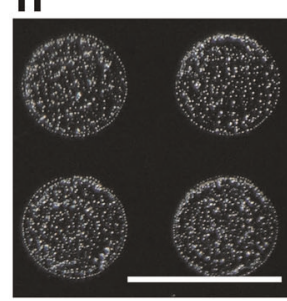

i b

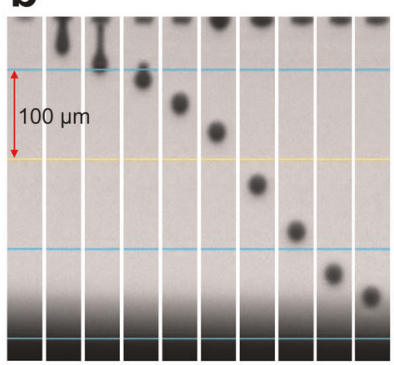

f


C

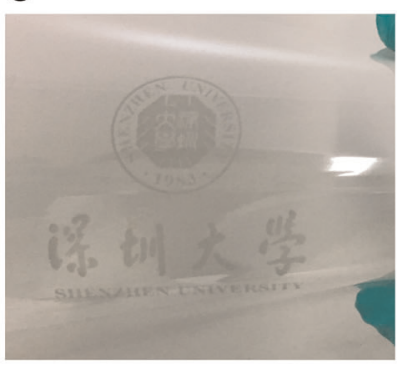

g

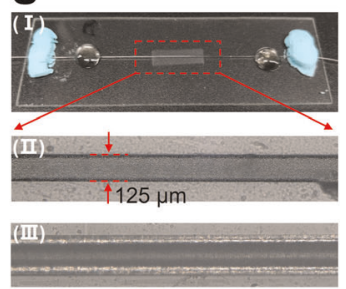

k

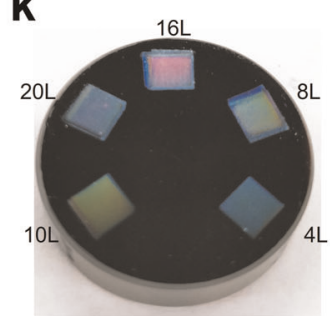

Fig. 2 Inkjet printing characterizations. a Inkjet printing schematic diagram. b Printing droplet sequences observed from the printer stroboscopic camera. c Inkjet printed logo of Shenzhen University on a transparent and flexible PET film. $\mathbf{d}-\mathbf{f}$ and $\mathbf{h}-\mathbf{j}$ The printed dots, stripes and plane on glass substrate, scale bar: $100 \mu \mathrm{m}$. g (I) The side-polished fiber saturable absorber. (II) Printed (12 layers) and (III) unprinted sidepolished fiber checked by an optical microscope. $\mathbf{k}$ Inkjet printed MXene saturable absorber mirror (SAM) with different printing layers. The clockwise printed layers: $10 \mathrm{~L}, 20 \mathrm{~L}, 16 \mathrm{~L}, 8 \mathrm{~L}, 4 \mathrm{~L}$

a

b

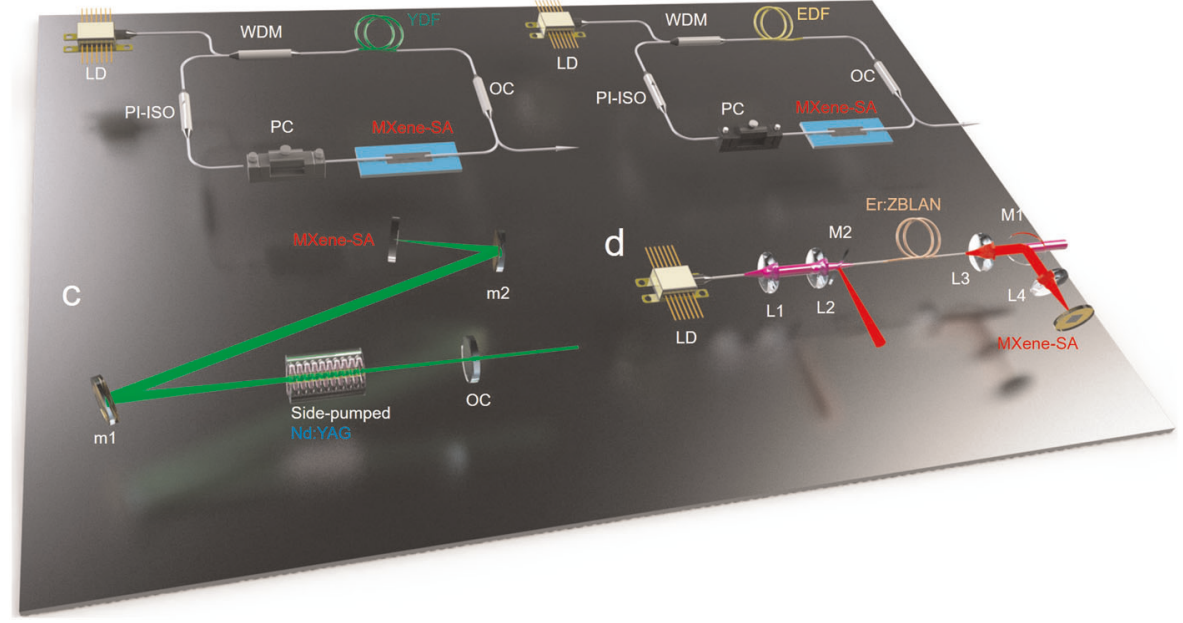

Fig. 3 Schematic diagrams of laser resonators. Laser diode pumped a Yb-doped and $\mathbf{b}$ Er-doped fiber laser resonators with an inkjet-printed MXene side-polished fiber saturable absorber. c Side-pumped Nd-YAG solid-state resonator, and d laser diode pumped Er-doped ZBLAN MIR fiber laser resonator with MXene printed on the end gold mirrors

The estimated intra-cavity net dispersion of Yb-doped and Erdoped are $0.227 \mathrm{ps}^{2}$ and $-0.22 \mathrm{ps}^{2}$, respectively. The length of the side-polished portion is $10 \mathrm{~mm}$, and the top surface has a close distance $(\sim 1 \mu \mathrm{m})$ to the fiber guiding core to achieve efficient evanescent field-MXene interactions. In order to completely overlap the side-polished fiber, the rectangularly printing area is
$3 \times 12 \mathrm{~mm}^{2}$. The MXene nanosheets are homogeneously printed on the side-polished fiber, as shown in Fig. $2 \mathrm{~g}$.

Compared to fiber resonator, all-solid-state resonator could be more challenge for free-space alignment and pulsed laser operation. Here, a side-pumped Nd:YAG solid-state laser resonator is conceived and constructed as shown in Fig. 3c. The size of $\mathrm{Nd}$ : 
a
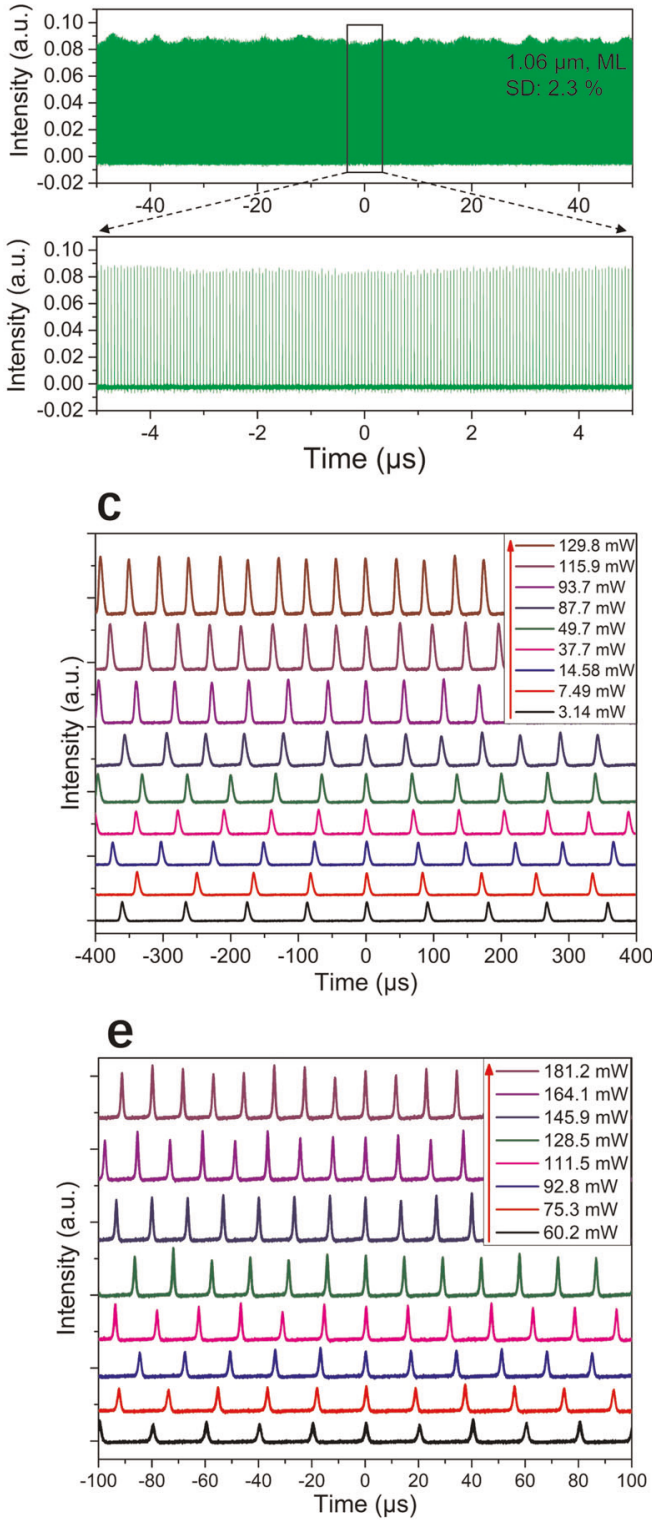

b
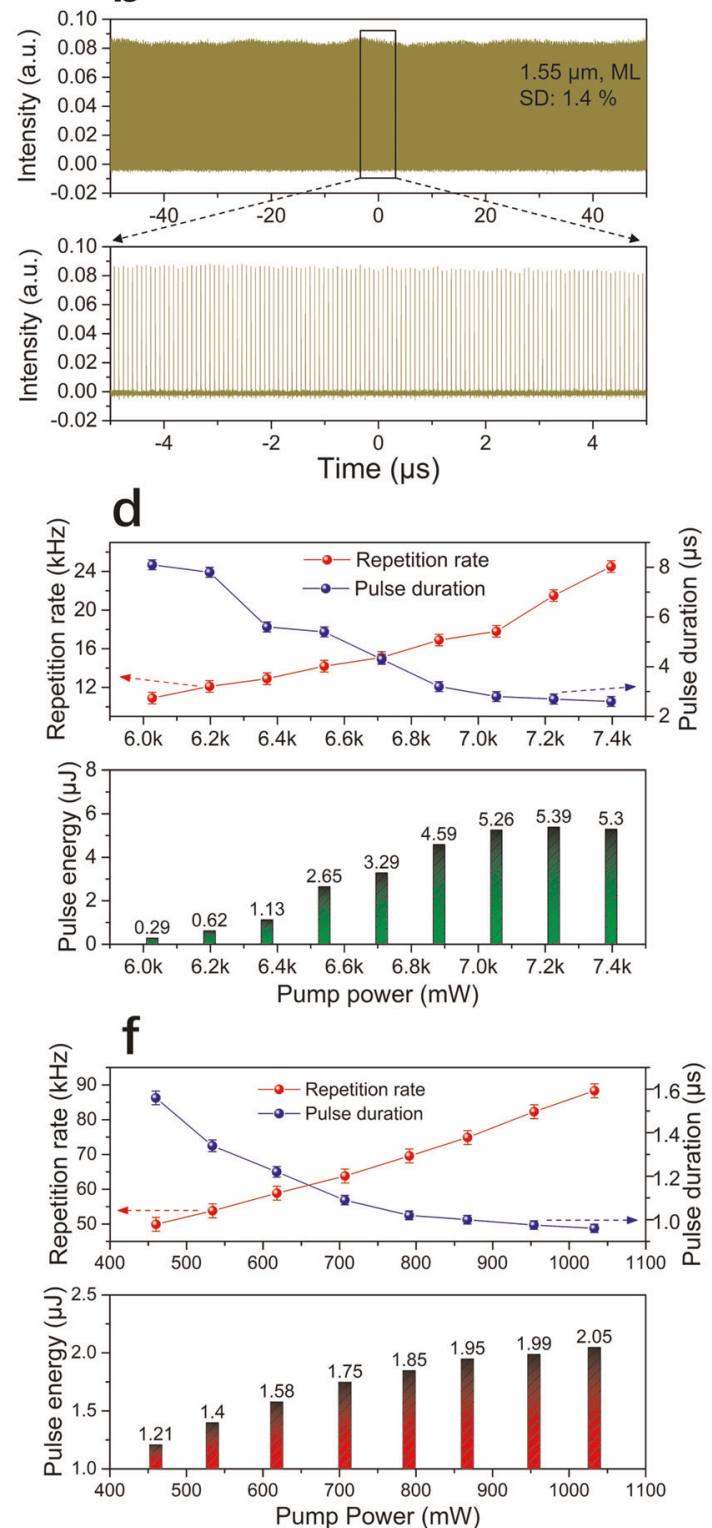

Fig. 4 Broadband pulsed laser operations. Oscilloscope spectrum of the phase locked pulse trains at $\mathbf{a} 1.06 \mu \mathrm{m}$ and $\mathbf{b} 1.55 \mu \mathrm{m}$. The modelocking pulse-to-pulse fluctuation is $2.3 \%$ and $1.4 \%$, respectively. SD stand deviation. Pulse trains of Q-switched laser at c $1.06 \mu \mathrm{m}$ and e $2.8 \mu \mathrm{m}$ with the corresponding output powers. Q-switching pulse duration and repetition rate (up) and pulse energy (down) as a function of the pump power at d $1.06 \mu \mathrm{m}$ and $\mathbf{f} 2.8 \mu \mathrm{m}$

YAG rod is $\$ 3 \times 65 \mathrm{~mm}$. The side-pump source (GTPC-75S, GT Optics Co. Ltd) can deliver pump power as high as $75 \mathrm{~W}$ at the operation current of $25 \mathrm{~A}$. Two concave mirrors with focal length of $250 \mathrm{~mm}(\mathrm{~m} 1)$ and $50 \mathrm{~mm}(\mathrm{~m} 2)$ are set up to achieve small beam profile on the printed MXene saturable absorber gold mirror. The output mirror has an output coupling efficiency of $20 \%$. The absorber mirror is mounted on a manually transform xyz-stage to optimize the laser performance.

To further expand the laser operation regime of $M X e n e$, a linear fiber resonator incorporating the prepared MXene saturable absorber mirror is set up for mid-infrared ultrashort pulse generation, as shown in Fig. $3 \mathrm{~d}$. The pump source is supplied by a commercial laser diode ( $30 \mathrm{~W}$, BTW Beijing Ltd) with wavelength center at $976 \mathrm{~nm}$. The laser is coupled out with a fiber pigtail with a core diameter of $105 \mu \mathrm{m}$ and numerical aperture (NA) of 0.15 . The pump light is collimated by a biconvex lens $\left(f_{1}=50 \mathrm{~mm}\right)$, then is focused into the gain fiber by the second biconvex lens $\left(f_{2}=\right.$
$75 \mathrm{~mm}$ ). The 3-meter double cladding Er:ZBLAN fiber (FiberLabs Inc.) was doped with an impurity concentration of 70,000 ppm. The core diameter of Er:ZBLAN fiber is $15 \mu \mathrm{m}$ and NA of 0.12 . The D-shape cladding has a diameter of $240 \times 260 \mu \mathrm{m}$ (short $\times$ long) with NA of 0.4 . To adjust the incident beam area thus the laser intensity on the MXene SAM and avoid the Fresnel refraction, a ZnSe aspherical lens pair $\left(f_{3}=f_{4}=12.7 \mathrm{~mm}\right.$, AR@976 nm, 2.7-3.7 $\mu \mathrm{m}$ ) is established. A $45^{\circ}$ dichroic mirror (M1) with high transparency at pump wavelength $(\mathrm{T}>95 \%)$ and high reflectivity at laser wavelength $(R>99 \%)$ is added to remove the pump light effects on the MXene SA mirror. The outcoupling end facet of Er: ZBLAN fiber was cut perpendicular to the fiber axis, with a Fresnel reflection of $4 \%$ as the laser feedback. While the other facet was cut with a small angle of 8 degrees to avoid parasitic oscillation. Another identical $45^{\circ}$ dichroic mirror (M2) was used to separate the pump and output light. 


\section{a}

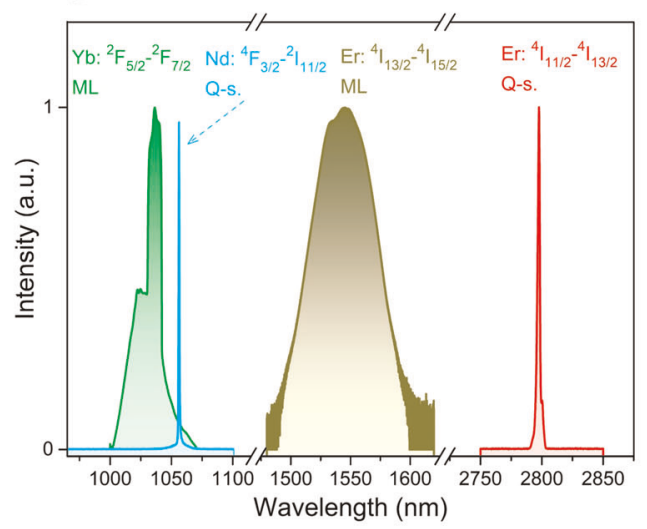

C
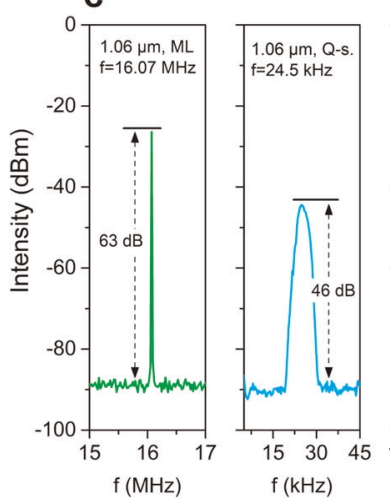

b

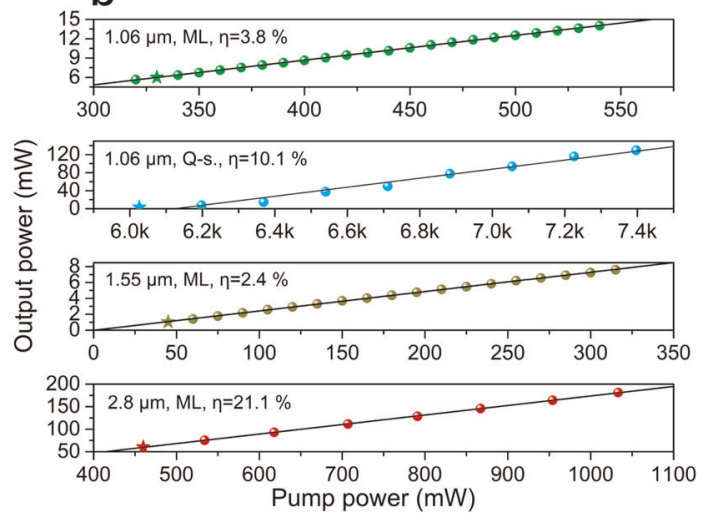

\section{d}
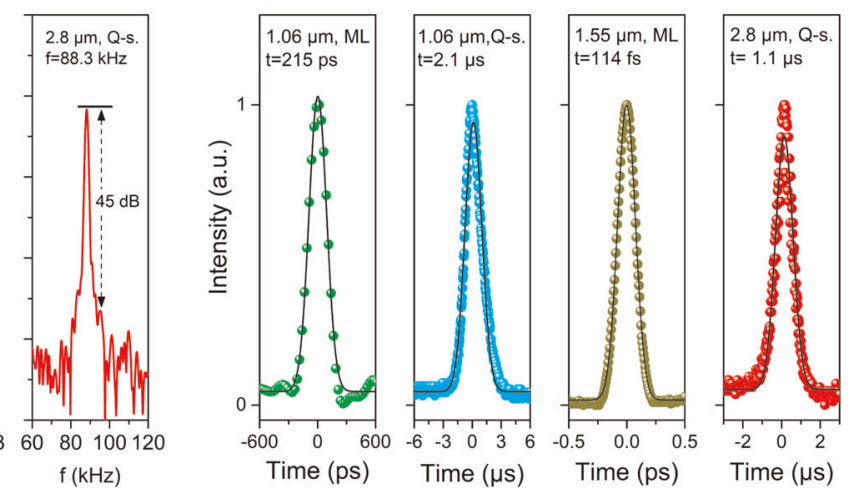

Fig. 5 Laser performance. a Laser operation wavelengths. The laser wavelength centers locate at $1034.5 \mathrm{~nm}\left(\mathrm{Yb}:{ }^{2} F_{5 / 2}{ }^{2} F_{7 / 2}\right), 1065.3 \mathrm{~nm}(\mathrm{Nd}$ : $\left.{ }^{4} F_{3 / 2}-{ }^{4} I_{11 / 2}\right), 1543.5 \mathrm{~nm}\left(\mathrm{Er}:{ }^{4} I_{13 / 2}-{ }^{4} I_{15 / 2}\right)$ and $2785.1 \mathrm{~nm}\left(\mathrm{Er}:{ }^{4} I_{11 / 2}-{ }^{4} I_{13 / 2}\right)$, respectively. ML: mode-locking, Q-s.: Q-switching. b The output power and slope efficiency. The fitted laser thresholds are $176 \mathrm{~mW}, 6124 \mathrm{~mW}, 68.9 \mathrm{~mW}$, and $178 \mathrm{~mW}$, respectively. The stars indicate the pulsed laser operation thresholds. c Radio frequency spectrum of pulse laser operations. $\mathbf{d}$ The output laser pulse durations. Gaussion fit is applied. Timebandwidth product (TBP) at $1.06 \mu \mathrm{m}$ and $1.55 \mu \mathrm{m}$ are estimated to be 391 and 0.367

Previous studies ${ }^{57,59}$ indicate that the nonlinear optical properties and linear losses of the saturable absorber can be finely tailored via directly varying the numbers of the printed layer. Here, fiber and gold mirror SAs with a range of printed layers are designed and fabricated. Stable continuous wave (CW) modelocked $(1.06$ and $1.55 \mu \mathrm{m})$ and Q-switched (1.06 and $2.8 \mu \mathrm{m})$ lasers with the spectral ranges spanning from near- to mid-infrared regimes have been realized, as shown in Fig. 4. The mode-locked pulse trains show a low peak-to-peak fluctuation ( 2\%) over a time of $100 \mu$ s for the two operation wavelength, while the Qswitched pulse trains show a slightly higher fluctuation of $\sim 5$ and $\sim 6 \%$ at $1.06 \mu \mathrm{m}$ and $2.8 \mu \mathrm{m}$, respectively. The high stability of the pulsed laser operations is confirmed via the high signal-to-noise ratio (SNR) as shown in Fig. $5 \mathrm{c}$. The repetition rate of phase-locking is a constant determined by the cavity length, to be $16.07 \mathrm{MHz}$ and $11.76 \mathrm{MHz}$, respectively. However, the repetition rate of the Qswitching monotonically increases from 10.9 to $24.5 \mathrm{kHz}$ at $1.06 \mu \mathrm{m}$, and 49.9 to $88.3 \mathrm{kHz}$ at $2.8 \mu \mathrm{m}$. Conversely, the pulse duration decreases from 8.1 to $2.6 \mu \mathrm{s}$ at $1.06 \mu \mathrm{m}$, and 1.56 to $1.1 \mu \mathrm{s}$ at $2.8 \mu \mathrm{m}$ in the meantime due to the increasing levels of saturation.

The laser wavelengths, output powers, repetition rates, and pulse durations of the laser operations in different resonators are summarized in Fig. 5. The steep spectral edges observed at the $1.06 \mu \mathrm{m}$ (Yb: ${ }^{2} F_{5 / 2}-{ }^{2} F_{7 / 2}$ ) is because of the normal dispersion mode-locking operation. The wide frequency band $(25.6 \mathrm{~nm}$ @3 dB) of Er: ${ }^{4} I_{13 / 2}-{ }^{4} I_{15 / 2}$ suggests the phase-locking can support an ultrashort temperal pulse $(\sim 114 \mathrm{fs})$ and is consistent with the autocorrelation characterization (APE pulseCheck 50), as shown in Fig. $5 \mathrm{~d}$ (see more information in $\mathrm{SI}$ ). As for the femtosecond laser pulse, the fiber pigtail with non-negligible dispersion can significantly affect the output pulse duration..$^{60,61}$ The fiber pigtail used here is a single-mode fiber with a length of $\sim 3 \mathrm{~m}$, and an estimated dispersion of $-0.065 \mathrm{ps}^{2}$. This indicates the output pulse experiences further compression during the output coupling. Different with the longitudinal mode phase-locked operation, the Q-switching operations show a relatively narrow spectrum band. A output slope efficiency as high as $21.1 \%$ (photonic efficiency of $60.5 \%)$ of the Q-switched mid-infrared operation was achieved. Noteworthy, all the pulsed laser operations can be initialized near the laser thresholds. The fundamental radio frequency is shown in Fig. 5c, high SNR radio frequency spectra can be observed from all the pulsed laser operations that confirms their high stability. The minimum pulse duration obtained at the highest pump level are $215 \mathrm{ps}, 2.6 \mu \mathrm{s}, 114 \mathrm{fs}$, and $1.1 \mu \mathrm{s}$, respectively. A comprehensive comparision of the laser performance with other reports using 2D materials-based saturable absorbers have been summaried in Tables S1-S4. Considering the maximum output power at the four wavelengths are $14.1 \mathrm{~mW}, 129.8 \mathrm{~mW}, 9.2 \mathrm{~mW}$ and $181.2 \mathrm{~mW}$, respectively, the achieved maximum pulse energies (peak power) are $0.88 \mathrm{~nJ}(4.1 \mathrm{~W}), 5.3 \mu \mathrm{J}(2.1 \mathrm{~W}), 0.78 \mathrm{~nJ}(6.86 \mathrm{~kW})$ and $2.1 \mu \mathrm{J}$ $(1.9 \mathrm{~W})$, which may suitable for a range of practical applications, e.g., mechanical machining, ${ }^{62}$ medical surgery, ${ }^{63}$ ecological monitoring, ${ }^{64}$ chemical reactions, ${ }^{65}$ and spectrocopies. ${ }^{66}$

We have described a full inkjet-printing approach for developing integrated broadband saturable absorbers via utilizing the distinct optical merits of 2D MXene. The well-controlled, cheap, binder-free and scalable ink formulation allows the agile integration of MXene small-footprint thin film patterns to variable setup geometrics. Ultrafast laser pulse generations were achieved 
spanning from $1 \mu \mathrm{m}$ to $3 \mu \mathrm{m}$ with operations modes of CW phaselocking and Q-switching in both fiber and solid-state resonators. The obtained shortest pulse duration is going to $100 \mathrm{fs}$ in the telecommunication window. Our demonstrates of versatile inkjetprinted MXene devices may pave the wave to the realizations of advanced MXene photonics devices shortly. More spectacularly, we hope this work could offer inspirations to explore other fullprint 2D crystals or heterostructures for integrated electrophotonics.

\section{DATA AVAILABILITY}

The data that support the findings of this study are available from the corresponding author upon reasonable request.

\section{ACKNOWLEDGEMENTS}

Financial support from the National Natural Science Foundation of China (Grant Nos. 61435010, 61575089, 61805146) and Science and Technology Innovation Commission of Shenzhen (Grant Nos. KQTD2015032416270385, JCYJ20150625103619275, JCYJ20170302153540973, and JCYJ20170412111625378), Science and Technology Planning Project of Guangdong Province (Grant No. 2016B050501005) and the Educational Commission of Guangdong Province (Grant No. 2016KCXTD006), Shenzhen-Hong Kong Innovation Cooperation Project (Grant No. SGLH20150205162842428), and the Science and Technology Development Fund (No. 007/2017/A1), Macao SAR, China are gratefully acknowledged.

\section{AUTHOR CONTRIBUTIONS}

H.Z. and X.J. conceived the project. X.J. and W.L. designed the experiments. X.J., W.L., T.H., and R.L. performed the experiments. Z.C. and C.L. instructed the inkjet printing experiments; Q.W. instructed the $1.06 \mu \mathrm{m}$ and $1.55 \mu \mathrm{m}$ fiber laser and $1.06 \mu \mathrm{m}$ solidstate laser experiments; G.X. instructed the $2.8 \mu \mathrm{m}$ MIR fiber laser experiments. All authors analyzed the data and discussed the experimental plans. X.J. wrote the manuscript and all authors commented and revised it. H.Z. supervised the whole project.

\section{ADDITIONAL INFORMATION}

Supplementary Information accompanies the paper on the npj $2 D$ Materials and Applications website (https://doi.org/10.1038/s41699-019-0117-3).

Competing interests: The authors declare no competing interests.

Publisher's note: Springer Nature remains neutral with regard to jurisdictional claims in published maps and institutional affiliations.

\section{REFERENCES}

1. Naguib, M. et al. Two-dimensional nanocrystals produced by exfoliation of $\mathrm{Ti}_{3} \mathrm{AlC}_{2}$. Adv. Mater. 23, 4248-4253 (2011).

2. Anasori, B., Lukatskaya, M. R. \& Gogotsi, Y. 2D metal carbides and nitrides (MXenes) for energy storage. Nat. Rev. Mater. 2, 16098 (2017).

3. Lin, H., Wang, X. G., Yu, L. D., Chen, Y. \& Shi, J. L. Two-dimensional ultrathin MXene ceramic nanosheets for photothermal conversion. Nano Lett. 17, 384-391 (2017).

4. Xue, Q. et al. Photoluminescent $\mathrm{Ti}_{3} \mathrm{C}_{2}$ MXene quantum dots for multicolor cellular imaging. Adv. Mater. 29, 6 (2017).

5. Shahzad, F. et al. Electromagnetic interference shielding with 2D transition metal carbides (MXenes). Science 353, 1137-1140 (2016).

6. Ran, J. R. et al. $\mathrm{Ti}_{3} \mathrm{C}_{2} \mathrm{MXene}$ co-catalyst on metal sulfide photo-absorbers for enhanced visible-light photocatalytic hydrogen production. Nat. Commun. 8, 10 (2017).

7. Li, R. Y., Zhang, L. B., Shi, L. \& Wang, P. MXene $\mathrm{Ti}_{3} \mathrm{C}_{2}$ : an effective $2 \mathrm{D}$ light-to-heat conversion material. Acs Nano 11, 3752-3759 (2017).

8. Jiang, $X$. et al. Broadband nonlinear photonics in few-layer $M X e n e \mathrm{Ti}_{3} \mathrm{C}_{2} \mathrm{~T}_{\mathrm{x}}(\mathrm{T}=\mathrm{F}$, O, or OH). Laser Photon. Rev. 12 (2018).

9. Zhang, X., Ma, Z. N., Zhao, X. D., Tang, Q. \& Zhou, Z. Computational studies on structural and electronic properties of functionalized MXene monolayers and nanotubes. J. Mater. Chem. A 3, 4960-4966 (2015).

10. Xie, Y. \& Kent, P. R. C. Hybrid density functional study of structural and electronic properties of functionalized $\mathrm{Ti}_{\mathrm{n}+1} \mathrm{X}_{\mathrm{n}}(\mathrm{X}=\mathrm{C}, \mathrm{N})$ monolayers. Phys. Rev. B 87, 10 (2013).
11. Lee, Y., Hwang, Y., Cho, S. B. \& Chung, Y. C. Achieving a direct band gap in oxygen functionalized-monolayer scandium carbide by applying an electric field. Phys. Chem. Chem. Phys. 16, 26273-26278 (2014).

12. Balci, E., Akkus, U. O. \& Berber, S. Band gap modification in doped MXene: $\mathrm{Sc}_{2} \mathrm{CF}_{2}$. J. Mater. Chem. C 5, 5956-5961 (2017).

13. Dong, L., Kumar, H., Anasori, B., Gogotsi, Y. \& Shenoy, V. B. Rational design of twodimensional metallic and semiconducting spintronic materials based on ordered double-transition-metal MXenes. J. Phys. Chem. Lett. 8, 422-428 (2017).

14. Lee, Y., Cho, S. B. \& Chung, Y. C. Tunable indirect to direct band gap transition of monolayer $\mathrm{Sc}_{2} \mathrm{CO}_{2}$ by the strain effect. Acs Appl. Mater. Interfaces 6, 14724-14728 (2014).

15. Hantanasirisakul, K. et al. Fabrication of $\mathrm{Ti}_{3} \mathrm{C}_{2} \mathrm{~T}_{\mathrm{x}} \mathrm{MXene}$ transparent thin films with tunable optoelectronic properties. Adv. Electron. Mater. 2, 7 (2016).

16. Ling, Z. et al. Flexible and conductive MXene films and nanocomposites with high capacitance. Proc. Natl Acad. Sci. USA 111, 16676-16681 (2014).

17. Nair, R. R. et al. Fine structure constant defines visual transparency of graphene. Science 320, 1308-1308 (2008).

18. Chaudhuri, K. et al. Highly broadband absorber using plasmonic titanium carbide (MXene). ACS Photonics 5, 1115-1122 (2018).

19. Jhon, Y. I. et al. Metallic MXene saturable absorber for femtosecond mode-locked lasers. Adv. Mater. 29 (2017).

20. Sun, $X$. et al. Few-layer $\mathrm{Ti}_{3} \mathrm{C}_{2} \mathrm{~T}_{\mathrm{x}}(\mathrm{Tx}=\mathrm{O}, \mathrm{OH}$, or $\mathrm{F}$ ) saturable absorber for a femtosecond bulk laser. Opt. Lett. 43, 3862-3865 (2018).

21. Tuo, M. et al. Ultrathin $2 \mathrm{D}$ transition metal carbides for ultrafast pulsed fiber lasers. ACS Photonics 5, 1808-1816 (2018).

22. Gogotsi, Y. CHEMICAL VAPOUR DEPOSITION Transition metal carbides go 2D. Nat. Mater. 14, 1079-1080 (2015).

23. Dua, V. et al. All-organic vapor sensor using inkjet-printed reduced graphene oxide. Angew. Chem. Int. Ed. 49, 2154-2157 (2010).

24. Shin, K.-Y., Hong, J.-Y. \& Jang, J. Micropatterning of graphene sheets by inkjet printing and its wideband dipole-antenna application. Adv. Mater. 23, 2113-2118 (2011).

25. Huang, L., Huang, Y., Liang, J., Wan, X. \& Chen, Y. Graphene-based conducting inks for direct inkjet printing of flexible conductive patterns and their applications in electric circuits and chemical sensors. Nano Res. 4, 675-684 (2011).

26. Le, L. T., Ervin, M. H., Qiu, H., Fuchs, B. E. \& Lee, W. Y. Graphene supercapacitor electrodes fabricated by inkjet printing and thermal reduction of graphene oxide. Electrochem. Commun. 13, 355-358 (2011).

27. Shin, K.-Y., Hong, J.-Y. \& Jang, J. Flexible and transparent graphene films as acoustic actuator electrodes using inkjet printing. Chem. Commun. 47, 8527-8529 (2011).

28. Zhang, L. et al. Inkjet printing high-resolution, large-area graphene patterns by coffee-ring lithography. Adv. Mater. 24, 436-440 (2012).

29. Torrisi, F. et al. Inkjet-printed graphene electronics. ACS nano 6, 2992-3006 (2012).

30. Li, J. et al. Efficient inkjet printing of graphene. Adv. Mater. 25, 3985-3992 (2013).

31. Withers, F. et al. Heterostructures produced from nanosheet-based inks. Nano Lett. 14, 3987-3992 (2014).

32. Secor, E. B., Prabhumirashi, P. L., Puntambekar, K., Geier, M. L. \& Hersam, M. C. Inkjet printing of high conductivity, flexible graphene patterns. J. Phys. Chem. Lett. 4, 1347-1351 (2013).

33. Secor, E. B., Ahn, B. Y., Gao, T. Z., Lewis, J. A. \& Hersam, M. C. Rapid and versatile photonic annealing of graphene inks for flexible printed electronics. Adv. Mater. 27, 6683-6688 (2015).

34. Kim, J. et al. Direct exfoliation and dispersion of two-dimensional materials in pure water via temperature control. Nat. Commun. 6, 8294 (2015).

35. Santra, S. et al. CMOS integration of inkjet-printed graphene for humidity sensing. Sci. Rep. 5, 17374 (2015).

36. Dodoo-Arhin, D. et al. Inkjet-printed graphene electrodes for dye-sensitized solar cells. Carbon 105, 33-41 (2016).

37. $\mathrm{Li}$, J. et al. Scalable fabrication and integration of graphene microsupercapacitors through full inkjet printing. ACS nano 11, 8249-8256 (2017).

38. Finn, D. J. et al. Inkjet deposition of liquid-exfoliated graphene and $\mathrm{MoS}_{2}$ nanosheets for printed device applications. J. Mater. Chem. C 2, 925-932 (2014).

39. Kelly, A. G. et al. All-printed thin-film transistors from networks of liquid-exfoliated nanosheets. Science 356, 69-73 (2017).

40. Kelly, A. G., Finn, D., Harvey, A., Hallam, T. \& Coleman, J. N. All-printed capacitors from graphene-BN-graphene nanosheet heterostructures. Appl. Phys. Lett. 109, 023107 (2016).

41. Carey, T. et al. Fully inkjet-printed two-dimensional material field-effect heterojunctions for wearable and textile electronics. Nat. Commun. 8, 1202 (2017).

42. McManus, D. et al. Water-based and biocompatible 2D crystal inks for all-inkjetprinted heterostructures. Nat. Nanotechnol. 12, 343-350 (2017).

43. Li, J., Naiini, M. M., Vaziri, S., Lemme, M. C. \& Östling, M. Inkjet printing of $\mathrm{MoS}_{2}$. Adv. Funct. Mater. 24, 6524-6531 (2014). 
44. Hu, G. et al. Black phosphorus ink formulation for inkjet printing of optoelectronics and photonics. Nat. Commun. 8 (2017).

45. Aleeva, Y. \& Pignataro, B. Recent advances in upscalable wet methods and ink formulations for printed electronics. J. Mater. Chem. C 2, 6436-6453 (2014).

46. Reis, N. \& Derby, B. Inkjet deposition of ceramic suspensions: modeling and experiments of droplet formation. MRS Online Proceedings Library Archive 625 (2000).

47. Vural, M. et al. Inkjet printing of self-assembled 2D titanium carbide and protein electrodes for stimuli-responsive electromagnetic shielding. Adv. Funct. Mater. 28, 1801972 (2018).

48. Shi, C. Y. et al. Structure of nanocrystalline $\mathrm{Ti}_{3} \mathrm{C}_{2}$ MXene using atomic pair distribution function. Phys. Rev. Lett. 112, 5 (2014).

49. Van Osch, T. H., Perelaer, J., de Laat, A. W. \& Schubert, U. S. Inkjet printing of narrow conductive tracks on untreated polymeric substrates. Adv. Mater. 20, 343-345 (2008)

50. Derby, B. Inkjet printing of functional and structural materials: fluid property requirements, feature stability, and resolution. Annu. Rev. Mater. Res. 40, 395-414 (2010).

51. Ying, Y. et al. Two-dimensional titanium carbide for efficiently reductive removal of highly toxic chromium(VI) from water. ACS Appl. Mater. Interfaces 7, 1795-1803 (2015).

52. Shijian, L., Harris, T. \& Wong, C. P. in Proceedings International Symposium on Advanced Packaging Materials Processes, Properties and Interfaces (IEEE Cat. No.01TH8562). 299-304.

53. Papakonstantinou, D., Amanatides, E., Mataras, D., loannidis, V. \& Nikolopoulos, P. Improved surface energy analysis for plasma treated PET films. Plasma Process. Polym. 4, S1057-S1062 (2007).

54. Hu, H. \& Larson, R. G. Marangoni effect reverses coffee-ring depositions. J. Phys. Chem. B 110, 7090-7094 (2006).

55. Yin, Z., Huang, Y., Bu, N., Wang, X. \& Xiong, Y. Inkjet printing for flexible electronics: materials, processes and equipments. Chin. Sci. Bull. 55, 3383-3407 (2010).

56. Keller, U. et al. Semiconductor saturable absorber mirrors (SESAM's) for femtosecond to nanosecond pulse generation in solid-state lasers. IEEE J. Sel. Top. Quantum Electron. 2, 435-453 (1996).

57. Jiang, X. et al. Low-dimensional nanomaterial saturable absorbers for ultrashortpulsed waveguide lasers. Opt. Mater. Express 8, 3055-3071 (2018).

58. Jiang, $X$. et al. Epsilon-near-zero medium for optical switches in a monolithic waveguide chip at $1.9 \mu \mathrm{m}$. Nanophotonics 7, 1835 (2018).
59. Jiang, X. et al. Bismuth telluride topological insulator nanosheet saturable absorbers for q-switched mode-locked Tm:ZBLAN waveguide lasers. Ann. der Phys. 528, 543-550 (2016).

60. Tamura, K., Ippen, E. P., Haus, H. A. \& Nelson, L. E. 77-fs pulse generation from a stretched-pulse mode-locked all-fiber ring laser. Opt. Lett. 18, 1080-1082 (1993).

61. Haus, H. A., Tamura, K., Nelson, L. E. \& Ippen, E. P. Stretched-pulse additive pulse mode-locking in fiber ring lasers: theory and experiment. IEEE J. Quantum Electron. 31, 591-598 (1995).

62. Gattass, R. R. \& Mazur, E. Femtosecond laser micromachining in transparent materials. Nat. Photonics 2, 219-225 (2008).

63. Jean, B. \& Bende, T. in Solid-State Mid-Infrared Laser Sources Vol. 89 Topics in Applied Physics (eds Irina T. Sorokina \& Konstantin L. Vodopyanov) Ch. 12, 530-565 (Springer Berlin Heidelberg, 2003).

64. Vasil'ev, B. I. \& Oussama, M. IR differential-absorption lidars for ecological mon itoring of the environment. Quantum Electron. 36, 801 (2006).

65. Zewail, A. H. Femtochemistry: atomic-scale dynamics of the chemical bond. J. Phys. Chem. A 104, 5660-5694 (2000).

66. Popmintchev, T. et al. Bright coherent ultrahigh harmonics in the keV X-ray regime from mid-infrared femtosecond lasers. Science 336, 1287-1291 (2012).

67. Kong, D., Le, L. T., Li, Y., Zunino, J. L. \& Lee, W. Temperature-dependent electrical properties of graphene inkjet-printed on flexible materials. Langmuir $\mathbf{2 8}$ 13467-13472 (2012).

68. Zhang, C. et al. Additive-free MXene inks and direct printing of microsupercapacitors. Nat. Commun. 10, 1795 (2019).

Open Access This article is licensed under a Creative Commons Attribution 4.0 International License, which permits use, sharing, adaptation, distribution and reproduction in any medium or format, as long as you give appropriate credit to the original author(s) and the source, provide a link to the Creative Commons license, and indicate if changes were made. The images or other third party material in this article are included in the article's Creative Commons license, unless indicated otherwise in a credit line to the material. If material is not included in the article's Creative Commons license and your intended use is not permitted by statutory regulation or exceeds the permitted use, you will need to obtain permission directly from the copyright holder. To view a copy of this license, visit http://creativecommons. org/licenses/by/4.0/.

(c) The Author(s) 2019 